Pure and Applied Mathematics Quarterly

Volume 7, Number 1

(Special Issue: In honor of

Frederick W. Gehring, Part 1 of 2)

$1-18,2011$

\title{
Quasiconformal Maps and Substantial Boundary Points
}

\author{
J.M. Anderson and A. Hinkkanen \\ Dedicated to Professor Fred Gehring, in admiration
}

\begin{abstract}
Let $D$ be a bounded domain in $\mathbb{C}$ with $\zeta_{0} \in \partial D$. We say that $\zeta_{0}$ is a substantial boundary point of $D$ for the affine stretch $x+i y \mapsto K x+i y$, where $K>1$, if for every neighbourhood $U$ of $\zeta_{0}$ and for every component $V$ of $U \cap D$ with $\zeta_{0} \in \partial V$, the maximal dilatation of $f$ is at least $K$ for every quasiconformal map $f$ of $V$ such that $f(x+i y)=K x+i y$ for all $x+i y \in \partial V \cap \partial D$.

We give here a criterion for a point $\zeta_{0}$ to be a substantial boundary point for the affine stretch in $D$ - Theorem 1.1 below. This will depend on the "narrowness" of $D$ near $\zeta_{0}$ though the particular way that $D$ is narrow may vary, as we shall show.
\end{abstract}

Keywords: quasiconformal mappings, substantial boundary points, affine stretch.

\section{INTRODUCTION}

Let $\mathbb{D}$ denote the unit disk $\{z:|z|<1\}$ in the complex plane $\mathbb{C}$, with boundary $\mathbb{T}$. Let $D$ be an arbitrary simply connected domain in $\mathbb{C}$ other than $\mathbb{C}$ itself. The

Received January 26, 2007

2000 Mathematics Subject Classification. Primary 30C62.

This material is based upon work supported by the National Science Foundation under Grant No. 0457291. The first author acknowledges support from the Leverhulme Trust (U.K.). 
affine stretch of $D$,

$$
f_{K}: x+i y \mapsto K x+i y, \quad \text { where } K>1,
$$

is the simplest example of a non-conformal quasiconformal mapping of $D$ onto $f_{K}(D)$ with complex dilatation

$$
\mu\left(f_{K}\right) \equiv \frac{K-1}{K+1}
$$

Such a mapping induces a quasiconformal mapping of $\mathbb{D}$ onto itself given by

$$
\tilde{f}_{K}=\phi_{2} \circ f_{K} \circ \phi_{1}^{-1}
$$

where $\phi_{1}$ and $\phi_{2}$ are conformal mappings of $D$ and $f_{K}(D)$ onto $\mathbb{D}$, respectively.

Of course, $\tilde{f}_{K}$ induces a quasisymmetric boundary homeomorphism on $\mathbb{T}$. For the basic facts on quasiconformal mappings we refer to [6].

Suppose that $\zeta_{0} \in \partial D$ and write $B\left(\zeta_{0}, r\right)=\left\{w:\left|w-\zeta_{0}\right|<r\right\}$. Given a neighbourhood $U$ of $\zeta_{0}$ we let $U_{0}$ be a component of $D \cap U$ with $\zeta_{0} \in \partial U_{0}$. Let $f_{K}$ be the affine stretch restricted to $\partial U_{0} \cap \partial D$ and let $f$ be a quasiconformal mapping of $U_{0}$ with $f(x+i y)=f_{K}(x+i y)=K x+i y$ on $\partial U_{0} \cap \partial D$, with maximal dilatation $K(f)$.

We set

$$
H\left(\zeta_{0}\right)=H\left(\zeta_{0}, f_{K}\right)=\inf K(f)
$$

where the infimum runs over all neighbourhoods $U$ of $\zeta_{0}$ and all such quasiconformal mappings $f$. Clearly $H\left(\zeta_{0}\right) \leq K$. We say that $\zeta_{0}$ is a substantial boundary point of $D$ for $f_{K}$ if $H\left(\zeta_{0}\right)=K$. The same definition applies to the function $\tilde{f}_{K}$ restricted to $\mathbb{T}$. The function

$$
H\left(\zeta, \tilde{f}_{K}\right), \quad \zeta \in \mathbb{T},
$$

is, of course, in $L^{\infty}(\mathbb{T})$. Although it need not be continuous, it has been shown by Fehlmann [2] that it is upper semicontinuous and so attains its supremum on $\mathbb{T}$. There are interesting connections between substantial boundary points and degenerating Hamilton sequences. We refer to [4] for details.

Criteria have been obtained in terms of the smoothness of a boundary function for the function to have no substantial boundary points [2]. If the geometry of the domain close to the boundary point $\zeta_{0}$ is regular enough then $\zeta_{0}$ is not a substantial boundary point for the affine stretch. For example, if $D$ has a non-zero angle at $\zeta_{0}$ then this is the case (see [7], pp. 123-124). One can ask 
whether $\zeta_{0}$ is a substantial boundary point for the affine stretch provided that $D$ is sufficiently irregular at $\zeta_{0}$. Note that obviously the substantial boundary points for the affine stretch form a closed subset of $\partial D$ so that in order to find interesting criteria, it may be a good idea to look at isolated substantial boundary points. We give below a sufficient condition for $\zeta_{0}$ to be a substantial boundary point. This condition does not preclude the possibility of there being other substantial boundary points arbitrarily close to $\zeta_{0}$ but it does not require it either, as our examples will show.

We prove the following result. Recall that a ring domain is a doubly connected domain in $\mathbb{C}$, both of whose boundary components contain more than one point. If $R$ is a ring domain then there is a conformal map $\varphi$ of $R$ onto the annulus $\left\{z: 1<|z|<R_{1}\right\}$ for a unique $R_{1}>1$, and, as usual, we define the conformal module $M(R)$ of $R$ by $M(R)=\left(\log R_{1}\right) /(2 \pi)$. We denote the closed line segment joining the points $w_{1}$ and $w_{2}$ in $\mathbb{C}$ by $\left[w_{1}, w_{2}\right]$, and the open line segment joining $w_{1}$ and $w_{2}$ by $\left(w_{1}, w_{2}\right)$. The area of a set $E \subset \mathbb{C}$ is denoted by $|E|$, and the Euclidean diameter of $E$ is denoted by diam $E$.

Theorem 1.1. Let $D$ be a bounded domain in $\mathbb{C}$ with $\zeta_{0} \in \partial D$. Suppose that there is a neighbourhood $W$ of $\zeta_{0}$ such that $W \cap \partial D$ is a Jordan arc. Suppose further that $D$ has a sequence of subdomains $D_{n}$ for $n \geq 1$ such that

(i) $\zeta_{0} \in \partial D_{n}$ for all $n$;

(ii) $D_{n+1} \subset D_{n}$ for all $n$;

(iii) $a_{n}=\operatorname{diam} D_{n} \rightarrow 0$ as $n \rightarrow \infty$;

(iv) $\left(\partial D_{n}\right) \backslash(\partial D)=L_{n}$ is an open line segment of length $b_{n}$;

(v) $b_{n}^{2} /\left|D_{n}\right| \rightarrow 0$ as $n \rightarrow \infty$; and

(vi) there exist positive constants $C_{0}>1$ and $\delta_{0} \leq 1 / 10$, depending only on $D$ and independent of $n$, such that whenever the distinct points $c_{1}$ and $c_{2}$ lie in $L_{n}=\left(z_{1}, z_{2}\right)$ with $c_{1}$ between $z_{1}$ and $c_{2}$ and with

$$
\left|c_{1}-c_{2}\right|<\delta_{0} \min \left\{\left|c_{1}-z_{1}\right|,\left|c_{2}-z_{2}\right|\right\},
$$

and $c_{3}=\left(c_{1}+c_{2}\right) / 2, r=\min \left\{\left|c_{3}-z_{1}\right|,\left|c_{3}-z_{2}\right|\right\}$, and further $B^{\prime}$ is the component of $B\left(c_{3}, r\right) \cap D$ containing $c_{3}$, then

$$
M\left(B \backslash\left[c_{1}, c_{2}\right]\right) \leq C_{0} M\left(B^{\prime} \backslash\left[c_{1}, c_{2}\right]\right) .
$$


Then $\zeta_{0}$ is a substantial boundary point of $D$ for the affine stretch $f_{K}: x+i y \mapsto$ $K x+i y$ for each $K>1$.

The assumption of Theorem 1.1 that there is a neighbourhood $W$ of $\zeta_{0}$ such that $W \cap \partial D$ is a Jordan arc is imposed to ensure that the domains $D_{n}$ give us all the information that is necessary concerning the behaviour of $D$ close to $\zeta_{0}$. Clearly this assumption is satisfied, for example, if $D$ is a Jordan domain.

The assumptions of Theorem 1.1 mean that there is a sequence of short cross cuts $L_{n}$ tending to $\zeta_{0}$, such that $D$ is very narrow at these cross cuts. However, the particular way that $D$ is narrow may vary, as we now show by examples. The condition (v) means that a disk with diameter $L_{n}$ has a small area if compared to the area of $D_{n}$. The condition (vi) states that there is, in a sense, enough space in $D$ (also outside $D_{n}$ ) close to $L_{n}$. We could formulate geometric conditions guaranteeing that (vi) holds, for example by requiring that $D$ contains the disk with diameter $L_{n}$, but that would be more restrictive than necessary. Therefore we have opted for including the module condition that we actually use in the proof.

Examples. Let $P$ be a non-rectangular parallelogram with one pair of sides parallel to the $x$-axis and of length $a$ (we call them the upper and lower sides of $P$ ). Let the other pair of sides have length $s a$, where $s>0$. For $n \geq 1$, let $P_{n}$ be a parallelogram congruent to $P$ and with one pair of sides parallel to the $x$-axis and of length $\alpha_{n}$. Then the other pair of sides has length $s \alpha_{n}$. Suppose that the points on the upper side of $P_{n+1}$ have imaginary part at most that of those on the lower side of $P_{n}$. Let $L_{n}$ be an open segment of length $b_{n}$ contained in the upper side of $P_{n}$ for $n \geq 2$. We assume that the right end point of $L_{n}$ coincides with the right end point of the upper side of $P_{n}$. Let $D$ be a bounded Jordan domain formed by taking the parallelograms $P_{n}$ and joining each $P_{n}$ to $P_{n+1}$ by forming a connection between $L_{n+1}$ and a segment of length at most $c b_{n+1}$, for some positive absolute constant $c$, on the lower side of $P_{n}$. We assume that $\alpha_{n} \rightarrow 0$ monotonically and $\sum_{n} \alpha_{n}<+\infty$, so that the $P_{n}$ converge to a single point $\zeta_{0} \in \partial D$. Let $D_{n}$ be the natural subdomain of $D$ whose boundary contains $L_{n}$, with $\zeta_{0} \in \partial D_{n}$. Suppose that $b_{n} / \alpha_{n} \rightarrow 0$. Then also $b_{n}^{2} /\left|D_{n}\right| \rightarrow 0$, and $D$ satisfies the conditions of Theorem 1.1, possibly apart from (vi). However, it is easy to adjust the construction to make sure that also (vi) is satisfied. 
We now see that small modifications of this example would lead to others, still satisfying the conditions of Theorem 1.1, but in a different way. Above, the segment $L_{n}$ is horizontal. We could take $L_{n}$ to be part of the right hand side boundary segment of $P_{n}$. This would lead to the set $V_{n}$ considered in Section 6 being non-empty. Next, the examples would look somewhat different depending on whether $L_{n}$ is only a small part of the right hand side boundary segment of $P_{n}$, or almost all of it. The latter can still be compatible with the assumptions of Theorem 1.1 provided that $b_{n}$ is small compared to $a_{n}$ and $\sqrt{\left|D_{n}\right|}$. With a suitable connection of $P_{n}$ to $P_{n-1}$, condition (vi) of Theorem 1.1 can still be satisfied. These choices may vary with $n$, so we can obtain a domain that is narrow infinitely often but in somewhat different ways. Obviously we can construct $D$ so that $\zeta_{0}$ is the only substantial boundary point of $D$.

In Section 2 we obtain a lemma that shows how one can estimate the dilatation of a quasiconformal extension of the affine stretch even when there is part of the boundary of the domain where the boundary values are not given. This is done by adapting the usual length-area method used in the proof of the Main Inequality of Reich and Strebel [7].

In Section 3 we adopt notation to discuss the kind of domains that satisfy the assumptions of Theorem 1.1.

In Sections 4 and 5 we perform auxiliary estimates required to control the behaviour of the function on that part of the boundary where boundary values are not prescribed. This is based on estimating moduli of quadrilaterals and the well known connection between such moduli and geometric quantities.

In Section 6 we put our previous results together and prove Theorem 1.1. In the concluding Section 7 we make some general remarks on possible generalisations.

\section{Horizontal FOLIATIONS}

Before we get to the proof of Theorem 1.1, we make some preliminary observations. Here the notation is not restricted to that of Theorem 1.1.

Given a bounded domain $D$ and the affine stretch $f_{K}: x+i y \mapsto K x+i y$ of $D$ into $D_{1}$, say, we may consider the boundary correspondence $f_{K} \mid \partial D$ on $\partial D$ and ask for all possible extensions $f$ of $f_{K} \mid \partial D$ into $D$. This problem, in the general case, has been much studied, see, for example, [1], [3], [5], [7], [9], [10], [11], [12], 
[13]. We wish here to consider the extensions $f$, say, with $f=f_{K}$ on $\partial D \backslash L$ where $L$ is in some appropriate sense a small subset of $\partial D$.

Let $D$ be a bounded domain, and $L$ a small line segment contained in the boundary of $D$. So we are looking at those quasiconformal mappings $f$ of $D$ such that $f=f_{K}$ on $\partial D \backslash L$ and the values of $f$ on $L$ are not prescribed. If $L$ is horizontal we may foliate $D$ by horizontal segments whose end points are in $\partial D \backslash L$ so that $f=f_{K}$ at all these end points. If $f=f_{K}$ on all of $\partial D$ then $K(f) \geq K$. But since there is an exception on $L$ we can only assert that $K(f) \geq K_{1}$, where $K_{1}$ is a quantity which may be smaller than $K$ but will be close to $K$ if the length of $L$ is small.

To deal with the values of $f$ on $L$, we show by means of techniques employing estimates of modules of ring domains and the connection of such modules to distances that $f \mid L$ cannot deviate very far from $f_{K} \mid L$ if $L$ is short and $K(f) \leq K$. If $L$ is not horizontal, then we cannot foliate all of $D$ by horizontal segments such that $f=f_{K}$ at both end points of every segment. However we may still foliate a subset of $D$ in this way, namely, the set obtained from $D$ by removing the segments with one end point on $L$. Even in the part of $D$ that was so removed, it is possible to emulate the standard arguments by again using the fact that $f \mid L$ will be close to $f_{K} \mid L$ if $K(f) \leq K$.

In the proof below, we will apply these ideas to the domains $D_{n}$ described in Theorem 1.1, and then the role of $L$ will be taken by $L_{n}$.

We introduce some standard notation. For a general quasiconformal mapping $f$ we write $f_{z}=\partial f / \partial z, f_{\bar{z}}=\partial f / \partial \bar{z}$. The quotient $f_{\bar{z}} / f_{z}$ is the complex dilatation $\mu_{f}$ and the Jacobian $J_{f}$ is $\left|f_{z}\right|^{2}-\left|f_{\bar{z}}\right|^{2}$. These are functions of $z$, but this dependence is frequently suppressed.

The following Lemma is based on an elementary application of the method of proof of the $M$-inequality due to Reich and Strebel, as given, for example, in [7], p. 110. It gets us started. Later, we will revisit the proof of this lemma to study a more general situation involving a non-horizontal line segment $L$.

Lemma 2.1. Let $D$ be a bounded open set in the plane. Let $f$ be a quasiconformal homeomorphism of $D$ such that for some $K>1$, we have $f=f_{K}$ on $\partial D \backslash L$ where $L \subset \partial D$ is a horizontal line segment. Then

$$
K^{2}|D| \leq K(f)|f(D)| .
$$


We assume in Lemma 2.1 that $D$ is an open set rather than a domain since we may wish to apply Lemma 2.1 to the situation where $D$ is obtained by removing a rectangle-like domain from a larger domain.

Lemma 2.1 gives a lower bound for $K(f)$ in terms of the quantity $|D| /|f(D)|$, which we should expect to be close to $1 / K$.

Proof of Lemma 2.1. For any $\eta \in \mathbb{R}$ let $\Gamma(\eta)=D \cap\{x+i \eta: x \in \mathbb{R}\}$. The set $\Gamma(\eta)$, if not empty, is the union of at most countably many open horizontal line segments. Each such segment has both of its boundary points in $\partial D \backslash L$. Let $L(\Gamma)$ denote the total length of paths comprising the set $\Gamma$.

Suppose that for a fixed $\eta$, the set $\Gamma(\eta)$ consists of only one segment. We write $\Gamma^{\prime}(\eta)=f_{K}(\Gamma(\eta)), \Gamma^{\prime \prime}(\eta)=f(\Gamma(\eta))$. Then $\Gamma^{\prime}(\eta)$ is a horizontal line segment with the same end points as the $\operatorname{arc} \Gamma^{\prime \prime}(\eta)$ so that $K L(\Gamma(\eta))=L\left(\Gamma^{\prime}(\eta)\right) \leq L\left(\Gamma^{\prime \prime}(\eta)\right)$. Since $f$ is absolutely continuous on lines, we can deduce that for almost every $\eta$

$$
\begin{aligned}
K \int_{\Gamma(\eta)} d x & \leq \int_{\Gamma(\eta)}\left|f_{z} d z+f_{\bar{z}} d \bar{z}\right| \\
& =\int_{\Gamma(\eta)}\left|f_{z}+f_{\bar{z}}\right| d x=\int_{\Gamma(\eta)}\left|f_{z}\right||1+\mu(f)| d x .
\end{aligned}
$$

If $\Gamma(\eta)$ consists of more than one segment, the above argument applies to each of them, and adding up we obtain the conclusion

$$
K \int_{\Gamma(\eta)} d x \leq \int_{\Gamma(\eta)}\left|f_{z}\right||1+\mu(f)| d x .
$$

for the entire set $\Gamma(\eta)$.

We note that

$$
\left|f_{z}\right|=\left(\frac{J_{f}}{1-\left|\mu_{f}\right|^{2}}\right)^{1 / 2}
$$

Integrating (2.1) with respect to $\eta$ between the limits arising from the bounded open set $D$ we obtain

$$
K|D| \leq \int_{D}\left(\frac{J_{f}}{1-\left|\mu_{f}\right|^{2}}\right)^{1 / 2}|1+\mu(f)| d x d y .
$$

Applying the Cauchy-Schwarz inequality we obtain

$$
K^{2}|D|^{2} \leq\left(\int_{D} J_{f} d x d y\right)\left(\int_{D} \frac{|1+\mu(f)|^{2}}{1-|\mu(f)|^{2}} d x d y\right) \leq|f(D)| K(f)|D| .
$$


This last inequality follows from the fact that

$$
\frac{|1+\mu(f)|^{2}}{1-|\mu(f)|^{2}} \leq \frac{(1+|\mu(f)|)^{2}}{1-|\mu(f)|^{2}}=\frac{1+|\mu(f)|}{1-|\mu(f)|} \leq K(f)
$$

almost everywhere in $D$. This proves Lemma 2.1 .

\section{The Set-up Related to the Domain $D$}

Let $D$ satisfy the assumptions of Theorem 1.1. Let $U$ be a neighbourhood of $\zeta_{0}$ and let $U_{0}$ be a component of $D \cap U$ with $\zeta_{0} \in \partial U_{0}$ such that $U \cap \partial D$ is a Jordan arc. Let $f$ be a quasiconformal map of $U_{0}$ with $f=f_{K}$ on $\left(\partial U_{0}\right) \cap(\partial D)$. There exists a positive integer $m$ such that $D_{m} \subset U_{0}$. We now view $f$ as a quasiconformal map of $D_{m}$. Then $f=f_{K}$ on $\partial D_{m} \backslash L_{m}$. To get a lower bound for $K(f)$, we may assume that $K(f) \leq K$.

We shall show that for all sufficiently large values of $m$, we have

$$
\frac{\left|f\left(D_{m}\right)\right|}{\left|D_{m}\right|} \leq K+\pi C_{1}^{2} \frac{b_{m}^{2}}{\left|D_{m}\right|}
$$

for a suitable positive constant $C_{1}$.

If $L_{m}$ were horizontal, we could combine this with Lemma 2.1 and obtain

$$
K(f) \geq K\left(1+\frac{\pi C_{1}^{2}}{K} \frac{b_{m}^{2}}{\left|D_{m}\right|}\right)^{-1} \rightarrow K \text { as } m \rightarrow \infty .
$$

However, since $L_{m}$ need not be horizontal, a more careful argument will be needed.

Clearly the part of $D$ which is far from $\zeta_{0}$ plays no role in the argument.

We discuss the above selection of $m$ more carefully. First note that $U_{0}$ contains $D \cap B\left(\zeta_{0}, r\right)$ for some $r>0$, and hence there exists $m_{1} \geq 2$ such that $D_{n} \subset U_{0}$ for all $n \geq m_{1}$. We choose $m>m_{1}$ such that if $D_{m}$ is the subdomain of $D$ which lies "below" the segment $L_{m}$ and $D_{m_{1}}$ is the subdomain of $D$ which lies "below" the segment $L_{m_{1}}$, then there exists $\rho>0$ such that $D_{m} \subset D \cap B\left(\zeta_{0}, \rho / 2\right)$ and $L_{m_{1}}$ lies outside $B\left(\zeta_{0}, \rho\right)$. It suffices to find a lower bound for $K\left(f \mid D_{m}\right)$. In what follows, $L_{m}$ need not be horizontal.

We denote the end points of $L_{m}$ by $z_{1}$ and $z_{2}$, respectively, suppressing the dependence on $m$. Recall that we denote the closed line segment joining the 
points $w_{1}$ and $w_{2}$ by $\left[w_{1}, w_{2}\right]$, and the open line segment joining $w_{1}$ and $w_{2}$ by $\left(w_{1}, w_{2}\right)$. Thus $L_{m}=\left(z_{1}, z_{2}\right)$.

The assumption that $D_{n} \subset U_{0}$ for all $n \geq m_{1}$ and not only for all $n \geq m$ means that there is some extra space in the domain of definition of $f$ "above" $D_{m}$. We will make some use of this later. Note that $D_{m} \subset D_{m_{1}}$, and $f$ is defined and is $K$-quasiconformal in $D_{m_{1}}$, with $f=f_{K}$ on $\partial D_{m_{1}} \cap \partial D$. The set $D_{m_{1}} \backslash D_{m}$ contains at least $D_{m-1} \backslash \overline{D_{m}}$.

Furthermore, by the assumption (vi) of Theorem 1.1, there exist fixed positive constants $C_{0}>1$ and $\delta_{0} \leq 1 / 10$, depending only on $D$ and independent of $m$, such that the following holds. Pick any points $c_{1} \in\left(z_{1}, z_{2}\right)=L_{m}$ and $c_{2} \in\left(c_{1}, z_{2}\right)$ such that

$$
\left|c_{1}-c_{2}\right|<\delta_{0} \min \left\{\left|c_{1}-z_{1}\right|,\left|c_{2}-z_{2}\right|\right\} .
$$

Recall from condition (vi) of Theorem 1.1 that $c_{3}=\left(c_{1}+c_{2}\right) / 2$ and $B=B\left(c_{3}, r\right)$ where $r=\min \left\{\left|c_{3}-z_{1}\right|,\left|c_{3}-z_{2}\right|\right\}$. We need not have $B \subset D$. Write $B^{\prime}$ for the component of $B \cap D$ containing $c_{3}$. Then clearly $\left(z_{1}, c_{2}\right) \subset B^{\prime}$ if $\left|c_{3}-z_{1}\right| \leq\left|c_{3}-z_{2}\right|$, and $\left(c_{1}, z_{2}\right) \subset B^{\prime}$ if $\left|c_{3}-z_{2}\right| \leq\left|c_{3}-z_{1}\right|$. Depending on the precise shape of $D$, it is conceivable that $B \backslash B^{\prime}$ could have two or more components.

Our assumption implies that

$$
M\left(B^{\prime} \backslash\left[c_{1}, c_{2}\right]\right) \leq M\left(B \backslash\left[c_{1}, c_{2}\right]\right) \leq C_{0} M\left(B^{\prime} \backslash\left[c_{1}, c_{2}\right]\right),
$$

where $M(R)$ denotes the module of the ring domain $R$.

Note that if $\infty \in \overline{f\left(D_{m_{1}}\right)}$ then there is a point $\zeta \in D_{m_{1}} \cup L_{m_{1}}$ such that $f(\zeta)=\infty$ (since the set $f\left(\partial D_{m_{1}} \cap \partial D\right)=f_{K}\left(\partial D_{m_{1}} \cap \partial D\right)$ is bounded). Taking $m_{1}$ larger, we manage to exclude $\zeta$ from $\overline{D_{m_{1}}}$. We assume that this has been done, if necessary. Thus we assume that $\overline{f\left(D_{m_{1}}\right)}$ is a compact subset of the finite complex plane $\mathbb{C}$.

\section{Auxiliary Results}

To use Lemma 2.1 we must find a lower bound for $\left|D_{m}\right| /\left|f\left(D_{m}\right)\right|$, or equivalently an upper bound for $\left|f\left(D_{m}\right)\right| /\left|D_{m}\right|$. For fixed $m$ we write $\gamma_{0}=\partial D_{m} \backslash L_{m}$. Then $f\left(D_{m}\right)$ is a Jordan domain with $\partial f\left(D_{m}\right)=f_{K}\left(\gamma_{0}\right) \cup f\left(L_{m}\right)$. If $f\left(D_{m}\right) \subset$ $f_{K}\left(D_{m}\right)$, we have $\left|f\left(D_{m}\right)\right| \leq\left|f_{K}\left(D_{m}\right)\right|=K\left|D^{\prime}\right|$. So we may assume that 
$f\left(D_{m}\right) \backslash f_{K}\left(D_{m}\right) \neq \emptyset$. Then we can find an upper bound for $\left|f\left(D_{m}\right)\right|$ by estimating

$$
s_{1}=\sup \left\{\left|f(z)-f\left(z_{1}\right)\right|: z \in L_{m}, f(z) \notin f_{K}\left(D_{m}\right)\right\} .
$$

Note that $f\left(z_{1}\right)=f_{K}\left(z_{1}\right)$ and $\operatorname{Im} f\left(z_{1}\right)=\operatorname{Im} z_{1}$. We clearly have $\left|f\left(D_{m}\right)\right| \leq$ $\left|f_{K}\left(D_{m}\right)\right|+\pi s_{1}^{2}$, and $\left|f_{K}\left(D_{m}\right)\right|=K\left|D_{m}\right|$.

The set $f\left(L_{m}\right) \backslash \overline{f_{K}\left(D_{m}\right)}$, possibly empty, is the union of at most countably many open arcs, $\cup_{k} \gamma_{k}$, say. We fix $k$ and suppress the dependence on $k$ in the notation. Then $\overline{\gamma_{k}}$ contains at least one point $w$ such that

$$
t=\left|f(w)-f\left(z_{1}\right)\right|=\max \left\{\left|f(z)-f\left(z_{1}\right)\right|: z \in \overline{\gamma_{k}}\right\} .
$$

If $f(w) \in\left[f_{K}\left(z_{1}\right), f_{K}\left(z_{2}\right)\right]$ then $t \leq K b_{m}$. Hence we may assume that $f(w) \notin$ $\overline{f_{K}\left(D_{m}\right)}$.

We require the following two results. The first one can be found in [6], Lemma 4.1, p. 23.

Lemma 4.1. Let $Q$ be a quadrilateral in the Riemann sphere $\overline{\mathbb{C}}$. Let $s_{a}=$ $s_{a}(Q)$ denote the distance of the a-sides of $Q$ measured in $Q$, that is, $s_{a}$ is the infimum of the lengths of closed Jordan arcs $\gamma$ such that $\gamma$ lies in the interior of $Q$ apart from its end points, and $\gamma$ has one end point on each of the a-sides of $Q$. Similarly let $s_{b}=s_{b}(Q)$ denote the distance of the $b$-sides of $Q$ measured in $Q$. Let $M(Q)$ denote the module of $Q$. Then

$$
M(Q) \leq \pi \frac{1+2 L}{L^{2}} \quad \text { where } \quad L=\log \left(1+\frac{2 s_{a}}{s_{b}}\right) .
$$

The conjugate quadrilateral $Q^{\prime}$ of $Q$ is obtained from $Q$ by interchanging the roles of the $a$-sides and $b$-sides. We have $M\left(Q^{\prime}\right)=1 / M(Q)$. Hence Lemma 4.1 implies that

$$
M(Q) \geq \frac{1}{\pi} \frac{\left(L^{\prime}\right)^{2}}{1+2 L^{\prime}} \quad \text { where } \quad L^{\prime}=\log \left(1+\frac{2 s_{b}(Q)}{s_{a}(Q)}\right) .
$$

The second result is known as Teichmüller's module theorem, and it can be found, e.g., in [6], p. 56 .

Lemma 4.2. If the ring domain $R$ separates the points 0 and $\zeta_{1}$ from $\zeta_{2}$ and $\infty$ then

$$
M(R) \leq 2 \mu\left(\sqrt{\frac{\left|\zeta_{1}\right|}{\left|\zeta_{1}\right|+\left|\zeta_{2}\right|}}\right) .
$$


Here, as usual, $\mu(r)=M(B(0,1) \backslash[0, r])$ is the module of the Grötzsch ring (see [6]). The function $\mu(r)$ is decreasing for $0<r<1$ and has an inverse function $\mu^{-1}(r)$ defined on $(0, \infty)$.

\section{Distance estimates}

Recall that $t$ is defined by (4.2) and that $\delta_{0}$ and $C_{0}$ are as in Section 3 (that is, as in Theorem 1.1, (vi)). We wish to show that there exists a positive constant $C_{1}$ depending on $K, \delta_{0}$ and $C_{0}$ alone, such that

$$
t \leq C_{1} b_{m} .
$$

Then $s_{1}$, defined by (4.1), also satisfies

$$
s_{1} \leq C_{1} b_{m} .
$$

We define $\delta_{1}$ and $\delta_{2}$ by

$$
\begin{aligned}
& \frac{2 \delta_{2}}{1+\delta_{2}^{2}}=\mu^{-1}\left(4 K C_{0} \mu\left(\frac{1}{4}\right)\right), \\
& \delta_{1}=\min \left\{\delta_{0}, \delta_{2}, \frac{1}{2}(\sqrt{e}-1)\right\} .
\end{aligned}
$$

Then $0<\delta_{2}<1$ and

$$
4 K C_{0} \mu\left(\frac{1}{4}\right)=\mu\left(\frac{2 \delta_{2}}{1+\delta_{2}^{2}}\right) \leq \mu\left(\frac{2 \delta_{1}}{1+\delta_{1}^{2}}\right) .
$$

We establish (5.1) with

$$
C_{1}=4 K \exp \left\{\frac{8 \pi^{2} K}{\delta_{1}}\right\}>4 K .
$$

We suppose now that (5.1) does not hold, where $C_{1}$ is defined by (5.3). Then there exists a first point $z_{3}$ on $\left(z_{1}, z_{2}\right)$ when moving from $z_{1}$ to $z_{2}$ such that $\left|f\left(z_{3}\right)-f\left(z_{1}\right)\right|=C_{1} b_{m}$. Similarly there is a last point $z_{4}$ on $\left(z_{1}, z_{3}\right)$ when moving from $z_{1}$ to $z_{3}$ such that $\left|f\left(z_{4}\right)-f\left(z_{1}\right)\right|=C_{1} b_{m} / 2$. Thus, for all $z$ on the segment $\left(z_{4}, z_{3}\right)$,

$$
\frac{C_{1} b_{m}}{2}<\left|f(z)-f\left(z_{1}\right)\right|<C_{1} b_{m}
$$

To get a contradiction we suppose that

$$
\left|z_{4}-z_{3}\right|<\delta_{1} \min \left\{\left|z_{4}-z_{1}\right|,\left|z_{2}-z_{3}\right|\right\}
$$


and define $B$ and $B^{\prime}$ as in (3.2) using $z_{4}$ and $z_{3}$ instead of $c_{1}$ and $c_{2}$. Now, of course, $f$ is $K$-quasiconformal in $B^{\prime}$ and so, since $\delta_{1} \leq \delta_{0}$, we obtain from (3.2) that

$$
M\left(B \backslash\left[z_{4}, z_{3}\right]\right) \leq C_{0} M\left(B^{\prime} \backslash\left[z_{4}, z_{3}\right]\right) \leq K C_{0} M\left(f\left(B^{\prime}\right) \backslash f\left(\left[z_{4}, z_{3}\right]\right)\right) .
$$

Now $\partial B^{\prime}$ contains at least one of the points $z_{1}$ and $z_{2}$ so that $\partial f\left(B^{\prime}\right)$ contains at least one of the points $f\left(z_{1}\right)$ and $f\left(z_{2}\right)$. We denote such a point by $b$, so $b \in\left\{f\left(z_{1}\right), f\left(z_{2}\right)\right\}$. Since $f\left(B^{\prime}\right)$ is bounded, the ring domain $f\left(B^{\prime}\right) \backslash f\left(\left[z_{4}, z_{3}\right]\right)$ separates the points $f\left(z_{3}\right)$ and $f\left(z_{4}\right)$ from the points $b$ and $\infty$. It follows from Lemma 4.2 and the invariance of the module under Möbius transformations that

$$
M\left(f\left(B^{\prime}\right) \backslash f\left(\left[z_{4}, z_{3}\right]\right)\right) \leq 2 \mu\left(r_{0}\right)
$$

where

$$
r_{0}=\left\{1+\left|\frac{b-f\left(z_{4}\right)}{f\left(z_{3}\right)-f\left(z_{4}\right)}\right|\right\}^{-1 / 2}
$$

To get a lower bound for $r_{0}$, we next obtain an upper bound for

$$
\left|\frac{b-f\left(z_{4}\right)}{f\left(z_{3}\right)-f\left(z_{4}\right)}\right| \text {. }
$$

Since $\left|f\left(z_{3}\right)-f\left(z_{1}\right)\right|=C_{1} b_{m}$ and $\left|f\left(z_{4}\right)-f\left(z_{1}\right)\right|=C_{1} b_{m} / 2$, it follows that $\left|f\left(z_{3}\right)-f\left(z_{4}\right)\right| \geq C_{1} b_{m} / 2$.

If $b=f\left(z_{1}\right)$ then $\left|b-f\left(z_{4}\right)\right|=\left|f\left(z_{1}\right)-f\left(z_{4}\right)\right|=C_{1} b_{m} / 2$, so that

$$
\left|\frac{b-f\left(z_{4}\right)}{f\left(z_{3}\right)-f\left(z_{4}\right)}\right| \leq 1
$$

If $b=f\left(z_{2}\right)$ then $\left|b-f\left(z_{4}\right)\right|=\left|f\left(z_{2}\right)-f\left(z_{4}\right)\right| \leq\left|f\left(z_{2}\right)-f\left(z_{1}\right)\right|+\left|f\left(z_{1}\right)-f\left(z_{4}\right)\right| \leq$ $K b_{m}+C_{1} b_{m} / 2$. Hence

$$
\left|\frac{b-f\left(z_{4}\right)}{f\left(z_{3}\right)-f\left(z_{4}\right)}\right| \leq \frac{K b_{m}+C_{1} b_{m} / 2}{C_{1} b_{m} / 2}=\frac{2 K+C_{1}}{C_{1}}=1+\frac{2 K}{C_{1}} \leq \frac{3}{2}
$$

by (5.3).

Thus in all cases

$$
r_{0} \geq\{1+3 / 2\}^{-1 / 2}=\sqrt{\frac{2}{5}}>\frac{1}{2} .
$$


On the other hand, considering a Möbius transformation taking $B \backslash\left[z_{4}, z_{3}\right]$ onto $B(0,1) \backslash\left[0, r_{1}\right]$, we find that

$$
M\left(B \backslash\left[z_{4}, z_{3}\right]\right) \geq \mu\left(r_{1}\right)
$$

where

$$
r_{1}=\frac{2 \delta_{1}}{1+\delta_{1}^{2}}
$$

Hence, by (5.2),

$$
4 K C_{0} \mu(1 / 4) \leq \mu\left(r_{1}\right)<2 K C_{0} \mu(1 / 4),
$$

which gives a contradiction. We deduce that (5.1) cannot hold, and so necessarily

$$
\left|z_{4}-z_{3}\right| \geq \delta_{1} \min \left\{\left|z_{4}-z_{1}\right|,\left|z_{2}-z_{3}\right|\right\} .
$$

Now let $Q$ be the quadrilateral whose domain is $D_{m}$ and whose $a$-sides are the arcs of $\partial D_{m}$ going along $\partial D_{m} \backslash L_{m}$ from $z_{1}$ to $z_{2}$ and along the segment $L_{m}$ from $z_{3}$ to $z_{4}$. Then we have (noting that $s_{a}(Q)$ is defined by means of distances measured inside $Q$ )

$$
s_{a}(Q) \leq \min \left\{\left|z_{4}-z_{1}\right|,\left|z_{2}-z_{3}\right|\right\}
$$

and $s_{b}(Q)=\left|z_{3}-z_{4}\right|$. Hence

$$
L_{1}=\log \left(1+\frac{2 s_{b}(Q)}{s_{a}(Q)}\right) \geq \log \left(1+2 \delta_{1}\right)=P_{1},
$$

say. Since $\delta_{1} \leq(1 / 2)(\sqrt{e}-1)$ by $(5.2)$ we see that $P_{1} \leq 1 / 2$. Thus, from (4.4),

$$
M(Q) \geq \frac{1}{\pi} \frac{P_{1}^{2}}{1+2 P_{1}} \geq \frac{P_{1}^{2}}{2 \pi} \geq \frac{\delta_{1}^{2}}{2 \pi} .
$$

Analogously, we write

$$
P_{2}=\log \left(1+\frac{2 s_{a}(f(Q))}{s_{b}(f(Q))}\right)
$$

so that, by (4.3),

$$
\frac{M(Q)}{K} \leq M(f(Q)) \leq \pi \frac{1+2 P_{2}}{P_{2}^{2}}
$$

We have $s_{b}(f(Q)) \leq K\left|z_{1}-z_{2}\right|=K b_{m}$ and $s_{a}(f(Q)) \geq C_{1} b_{m} / 2-K b_{m}$. Thus

$$
P_{2} \geq \log \left(1+\frac{\left(C_{1}-2 K\right) b_{m}}{2 K b_{m}}\right)=\log \left(1+\frac{C_{1}-2 K}{2 K}\right)=L_{0}
$$


say. Now, from (5.3), we have $\left(C_{1}-2 K\right) /(2 K)>1$ so that $L_{0} \geq \log 2>1 / 2$. Hence,

$$
\frac{M(Q)}{K} \leq M(f(Q)) \leq \pi \frac{1+2 L_{0}}{L_{0}^{2}} \leq \frac{4 \pi}{L_{0}}
$$

We deduce that

$$
\frac{4 \pi K}{L_{0}} \geq \frac{\delta_{1}^{2}}{2 \pi}
$$

and hence

$$
1+\frac{C_{1}-2 K}{2 K} \leq \exp \left\{\frac{8 \pi^{2} K}{\delta_{1}^{2}}\right\}
$$

which gives a contradiction to (5.3).

We conclude that $s_{1} \leq C_{1} b_{m}$.

We have proved that for all sufficiently large $m$, we have

$$
\frac{\left|f\left(D_{m}\right)\right|}{\left|D_{m}\right|} \leq \frac{\left|f_{K}\left(D_{m}\right)\right|+\pi s_{1}^{2}}{\left|D_{m}\right|} \leq K+\pi C_{1}^{2} \frac{b_{m}^{2}}{\left|D_{m}\right|} .
$$

This yields (3.1).

\section{Completion of the Proof of Theorem 1.1}

Choose $\varepsilon>0$. Since, by the assumptions of Theorem 1.1, we have that $b_{n}^{2} /\left|D_{n}\right| \rightarrow 0$, there is a positive integer $n_{0}$ such that for all $n \geq n_{0}$, we have

$$
b_{n}^{2} /\left|D_{n}\right|<\varepsilon^{2} .
$$

Now pick $n$ with $n \geq n_{0}$. Our aim is to get a lower bound for $K(f)$, where $f$ is a quasiconformal map of $U_{0}$ with $f=f_{K}$ on $\partial U_{0} \cap \partial D$ and hence on $\partial D_{n} \backslash L_{n}$, in terms of $\left|D_{n}\right|$ and $\left|f\left(D_{n}\right)\right|$. Note that $K(f) \geq K\left(f \mid D_{n}\right)$ for all large $n$. We begin by exploring the consequences of Lemma 2.1 .

Now foliate the domain $D_{n}$ by horizontal line segments. (Note that foliation by horizontal segments is necessary for us to be able to use the ideas of the proof of Lemma 2.1. This is because we are considering a horizontal stretch $f_{K}$.) Let $V_{n}$ denote the union of those open horizontal line segments with one end point in $L_{n}$. Then $V_{n}$ is either a domain or the empty set. Write $U_{n}=D_{n} \backslash \overline{V_{n}}$. Clearly $V_{n}=\emptyset$ if, and only if, $L_{n}$ is horizontal. Note that $L_{n} \subset D$.

If $V_{n}=\emptyset$, we may apply Lemma 2.1 to $D_{n}$ and obtain

$$
K^{2}\left|D_{n}\right| \leq K(f)\left|f\left(D_{n}\right)\right| \text {. }
$$


If there is a sequence of values of $n$ with $V_{n}=\emptyset$, we may apply the arguments of the previous sections to these $n$ (with $m$ there replaced by $n$ ), and conclude, using (3.1) and the assumption that $\lim _{k \rightarrow \infty} b_{k}^{2} /\left|D_{k}\right|=0$, that $K(f) \geq K$. Namely, given any neighbourhood $U_{0}$ of $\zeta_{0}$, we merely have to consider a value of $n$ that is large enough for all these conditions to hold, and such that $D_{n}$ is contained in $U_{0}$ by a wide margin as explained before. So we may now assume that for all large $n$, we have $V_{n} \neq \emptyset$.

Suppose next that for a certain large $n \geq n_{0}$, we have $V_{n} \neq \emptyset$. Applying Lemma 2.1 to the bounded open set $U_{n}$ we obtain

$$
K^{2}\left|U_{n}\right| \leq K(f)\left|f\left(U_{n}\right)\right|
$$

Next, at least one of the inequalities

$$
\frac{b_{n}^{2}}{\left|V_{n}\right|}<\varepsilon \quad \text { and } \quad \frac{\left|V_{n}\right|}{\left|D_{n}\right|}<\varepsilon
$$

must hold for this $n$, for if both of these inequalities fail, then

$$
\frac{b_{n}^{2}}{\left|D_{n}\right|}=\frac{b_{n}^{2}}{\left|V_{n}\right|} \frac{\left|V_{n}\right|}{\left|D_{n}\right|} \geq \varepsilon^{2}
$$

which is a contradiction. Note that it may depend on $n$ which one of these two inequalities holds.

Suppose that $V_{n} \neq \emptyset$ and $\left|V_{n}\right| /\left|D_{n}\right|<\varepsilon$. This is the easier case. Now, since $U_{n} \subset D_{n}$, we obtain from (6.1) that

$$
\frac{K^{2}}{K(f)} \leq \frac{\left|f\left(U_{n}\right)\right|}{\left|U_{n}\right|} \leq \frac{\left|f\left(D_{n}\right)\right|}{\left|D_{n}\right|-\left|V_{n}\right|}=\frac{\left|f\left(D_{n}\right)\right|}{\left|D_{n}\right|} \frac{\left|D_{n}\right|}{\left|D_{n}\right|-\left|V_{n}\right|} \leq \frac{\left|f\left(D_{n}\right)\right|}{(1-\varepsilon)\left|D_{n}\right|} .
$$

We may then assume that $n$ is so large that the arguments given in the previous sections for $D_{m}$ apply when $m$ is replaced by $n$. Then (3.1) implies that

$$
\frac{\left|f\left(D_{n}\right)\right|}{\left|D_{n}\right|} \leq K+\pi C_{1}^{2} \frac{b_{n}^{2}}{\left|D_{n}\right|} .
$$

Thus altogether

$$
\frac{K^{2}}{K(f)} \leq \frac{1}{1-\varepsilon}\left(K+\pi C_{1}^{2} \frac{b_{n}^{2}}{\left|D_{n}\right|}\right)
$$

that is,

$$
K(f) \geq(1-\varepsilon) K\left(1+\pi C_{1}^{2} \frac{b_{n}^{2}}{K\left|D_{n}\right|}\right)^{-1}
$$


It follows that if there is a sequence of positive integers $n$ tending to infinity such that for each such $n$, we have $V_{n} \neq \emptyset$ and $\left|V_{n}\right| /\left|D_{n}\right|<\varepsilon$, then $K(f) \geq K(1-\varepsilon)$.

Suppose then that for some $n$, we have $V_{n} \neq \emptyset$ and $b_{n}^{2} /\left|V_{n}\right|<\varepsilon$. Now apply the proof of Lemma 2.1 to $V_{n}$. Note that by (5.1), we have

$$
L(f(\Gamma(\eta))) \geq K L(\Gamma(\eta))-C_{1} b_{n}
$$

for each $\eta$. Here we observe that even if the set $\Gamma(\eta)$ were the union of more than one line segment, at most one of those segments can have an end point on $L_{n}$. Thus for almost every $\eta$,

$$
K L(\Gamma(\eta))-C_{1} b_{n} \leq \int_{\Gamma(\eta)} \frac{\sqrt{J_{f}}}{\sqrt{1-\left|\mu_{f}\right|^{2}}}\left|1+\mu_{f}\right| d x
$$

so that integration over the appropriate values of $\eta$ gives

$$
K\left|V_{n}\right|-C_{1} b_{n}^{2} \leq K\left|V_{n}\right|-C_{1} b_{n} h_{n} \leq \sqrt{K(f)\left|V_{n}\right|\left|f\left(V_{n}\right)\right|}
$$

where $h_{n}$ denotes the variation of the $\eta$-coordinate over $V_{n}$ so that $h_{n} \leq b_{n}$. We may assume that $\varepsilon<K / C_{1}$ and therefore $K\left|V_{n}\right|-C_{1} b_{n}^{2}>0$. This yields

$$
\left(K\left|V_{n}\right|-C_{1} b_{n}^{2}\right)^{2} \leq K(f)\left|V_{n}\right|\left|f\left(V_{n}\right)\right| .
$$

To get an upper bound for $\left|f\left(V_{n}\right)\right|$, we recall the arguments concerning $D_{m}$. Clearly (5.1) implies that $\left|f\left(V_{n}\right)\right| \leq K\left|V_{n}\right|+\pi C_{1} b_{n}^{2}$. Thus

$$
\left(K\left|V_{n}\right|-C_{1} b_{n}^{2}\right)^{2} \leq K(f)\left|V_{n}\right|\left(K\left|V_{n}\right|+\pi C_{1} b_{n}^{2}\right) .
$$

It follows that

$$
K(f) \geq \frac{\left(K\left|V_{n}\right|-C_{1} b_{n}^{2}\right)^{2}}{\left|V_{n}\right|\left(K\left|V_{n}\right|+\pi C_{1} b_{n}^{2}\right)}=K \frac{\left(1-\frac{C_{1}}{K} \frac{b_{n}^{2}}{\left|V_{n}\right|}\right)^{2}}{1+\frac{\pi C_{1}}{K} \frac{b_{n}^{2}}{\left|V_{n}\right|}} .
$$

This last expression is greater than or equal to

$$
K \frac{\left(1-\varepsilon \frac{C_{1}}{K}\right)^{2}}{1+\varepsilon \frac{\pi C_{1}}{K}} .
$$

Thus $K(f) \geq K\left(1-C^{\prime} \varepsilon\right)$, where $C^{\prime}>1$ depends on $K, \delta_{0}$, and $C_{0}$ only. Now, since for each $\varepsilon>0$, there are infinitely many $n$ for which $V_{n} \neq \emptyset$ and $b_{n}^{2} /\left|V_{n}\right|<\varepsilon$, or there are infinitely many $n$ for which $V_{n} \neq \emptyset$ and $\left|V_{n}\right| /\left|D_{n}\right|<\varepsilon$, it follows that $K(f) \geq K\left(1-C^{\prime} \varepsilon\right)$ for each $\varepsilon>0$. Thus $K(f) \geq K$, so that $\zeta_{0}$ is a substantial boundary point of $D$. This completes the proof of Theorem 1.1. 


\section{GENERAL REMARKS}

The result of Theorem 1.1 is, of course, very special but it raises the question of whether there would be results of the same type in more general situations. It would also seem reasonable to suppose that the line segment $L_{n}$ can be replaced by any Jordan arc, and that the condition $b_{n}^{2} /\left|D_{n}\right| \rightarrow 0$ as $n \rightarrow \infty$ should be replaced by a condition involving harmonic measure. One can also ask to what extent the condition (vi) of Theorem 1.1, guaranteeing that there is a certain amount of space in $D$ around $L_{n}$, could be relaxed. Indeed, it seems possible that a condition of narrowness of a domain $D$ at a point $\zeta_{0} \in \partial D$ might be sufficient to make this point a substantial boundary point for the affine stretch.

\section{REFERENCES}

[1] J.M. Anderson and A. Hinkkanen, Quadrilaterals and extremal quasiconformal extensions, Comment. Math. Helv. 70 (1995), 455-474.

[2] R. Fehlmann, Ueber extremale quasikonforme Abbildungen, Comment. Math. Helv. 56 (1981), 558-580.

[3] R. Fehlmann, Quasiconformal mappings with free boundary components, Ann. Acad. Sci. Fenn. Ser. A I Math. 7 (1982), 337-347.

[4] R. Fehlmann and K. Sakan, On the set of substantial boundary points for extremal quasiconformal mappings, Complex Variables Theory Appl. 6 (1986), 323-335.

[5] R.S. Hamilton, Extremal quasiconformal mappings with prescribed boundary values, Trans. Amer. Math. Soc. 138 (1969), 399-406.

[6] O. Lehto and K.I. Virtanen, Quasiconformal Mappings in the Plane, Springer, Berlin, 1973.

[7] E. Reich, Quasiconformal mappings of the disk with given boundary values, pp. 101-137 in Lecture Notes in Math. 505, Springer, Berlin, 1976.

[8] E. Reich, An approximation condition and extremal quasiconformal extension, Proc. Amer. Math. Soc. 125 (1997), 1479-1481.

[9] E. Reich and K. Strebel, On the extremality of certain Teichmüller mappings, Comment. Math. Helv. 45 (1970), 353-362.

[10] E. Reich and K. Strebel, Extremal quasiconformal mappings with given boundary values, pp. 375-391 in Contributions to Analysis, Academic Press, 1974.

[11] Yuliang Shen, A counterexample theorem in quasiconformal mapping, Science in China (Series A), 43 (2000), 929-936.

[12] K. Strebel, Zur Frage der Eindeutigkeit extremaler quasikonformer Abbildungen des Einheitskreises II, Comment. Math. Helv. 39 (1964), 77-89.

[13] K. Strebel, On the dilatation of extremal quasiconformal mappings of polygons, Comment. Math. Helv. 74 (1999), 143-149. 
J.M. Anderson

Department of Mathematics,

University College London,

London WC1E 6BT, U.K.
A. Hinkkanen
Department of Mathematics,
University of Illinois at Urbana-Champaign,
1409 West Green Street, Urbana, IL 61801. USA
E-mail: aimo@uiuc.edu 